Reza Daryaei, Montaser Bakroon, Daniel Aubram, Frank Rackwitz

\title{
Numerical Investigation of the Frequency Influence on Soil Characteristics During Vibratory Driving of Tubular Piles
}

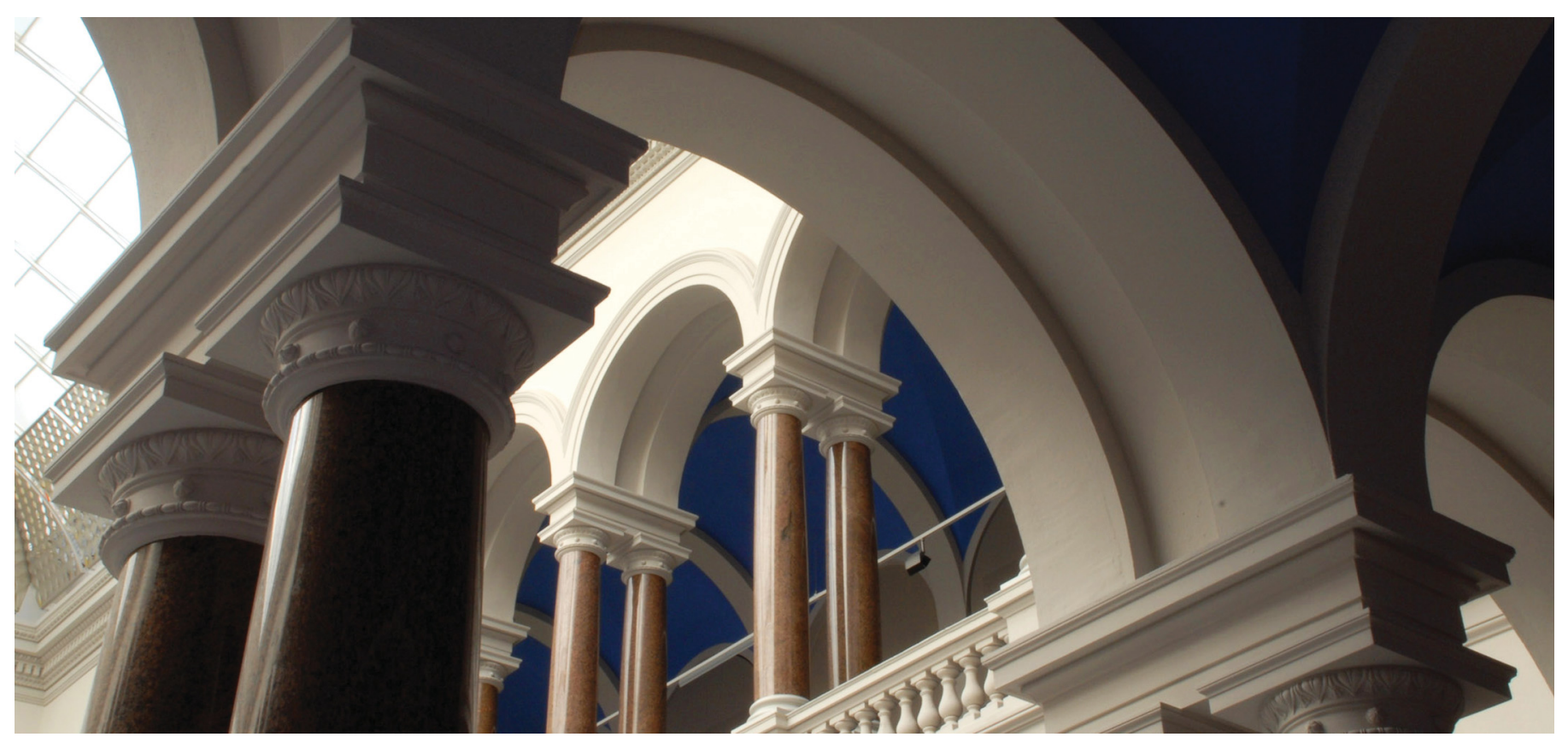

Daryaei, R., Bakroon, M., Aubram, D., \& Rackwitz, F. (2018). Numerical Investigation of the Frequency Influence on Soil Characteristics During Vibratory Driving of Tubular Piles. In: Shehata H., Desai C. (eds) Advances in Numerical Methods in Geotechnical Engineering. GeoMEast 2018. Sustainable Civil Infrastructures. Springer, Cham. pp. 48-61. https://doi.org/10.1007/978-3-030-01926-6_3 


\title{
Numerical investigation of the frequency influence on soil characteristics during vibratory driving of tubular piles
}

\author{
Reza Daryaei ${ }^{1}$, MSc, Montaser Bakroon ${ }^{1}$, MSc, Daniel Aubram ${ }^{1}$, Dr.-Ing., and Frank \\ Rackwitz ${ }^{1}$, Prof. Dr.-Ing. \\ 1 Chair of Soil Mechanics and Geotechnical Engineering, Technische Universität Berlin, Berlin, \\ Germany,r.daryaei@campus.tu-berlin.de
}

\begin{abstract}
In offshore geotechnics, tubular piles are commonly used as the foundation system. Such piles are installed using vibratory or impact driving. The choice of the proper loading configuration plays an important role in the driving performance, especially in reaching the desired penetration depth. Numerical evaluation of such processes involves handling large material deformation, making it hard for the classical numerical methods to reach a reliable result after significant deformation. In addition, in case of the dynamic cyclic loading, the soil exhibits complex behavior which emphasizes the role of a suitable soil constitutive equation. In this study, a numerical model is developed and utilized to evaluate the effects of the frequency in the vibratory installation of tubular piles on the neighboring soil. The numerical model employs the robust Multi-Material Arbitrary Lagrangian-Eulerian (MMALE) method in conjunction with an advanced material model formulation based on the hypoplasticity concept, and is validated against an experiment done at TU Berlin. Subsequently, a parametric study is performed by applying six different frequencies between 12 and $30 \mathrm{~Hz}$ to the dynamic load. The resulting penetration depth, void ratio and the lateral stress distribution in the soil are compared and evaluated. It is concluded that an optimum frequency must be determined to reach the maximum penetration depth by using the same load magnitudes.
\end{abstract}

\section{INTRODUCTION}

Tubular piles are one of the most-practiced types of deep foundation systems, especially for offshore applications. The installation performance of such piles is influenced by many factors, such as pile diameter and length, soil strength, pile-soil interaction, and the driving method. Conventionally, impact or vibratory driving is applied. Compared to impact driving, the vibratory driving produces less noise level, induce less damage to the pile, and offers a significantly higher penetration rates (Holeyman and Whenham 2017; Rausche 2002).

In this study, the effects of vibratory driving on the soil are investigated by varying the installation frequency. The soil response is analyzed by evaluating the resulting void ratio and lateral stress distribution. Due to the complex soil behavior and the large soil deformations during installation, a suitable numerical formulation is required to avoid the inevitable inaccuracies due to mesh distortion. Therefore, the Multi-Material Arbitrary Lagrangian-Eulerian (MMALE) method is employed to address this issue (Aubram et al. 2017; Benson 1992). In addition, owing to the high dependency of the numerical model accuracy on the soil material model, a robust constitutive equation based on the hypoplasticity concept is adopted (von Wolffersdorff 1996). There are numerous examples of successful simulations of complex problems using the MMALE 
and the hypoplastic material model in the literature (M. Bakroon et al. 2018a).

Previous works regarding vibratory driven piles consist of both numerical and experimental studies. One of the early studies on penetration rate was done by Feng and Deschamps (2000), where they derived an empirical equation for a penetration rate of a pile which is installed using hammer driving. In this equation, various effective factors on pile driving were considered.

In a series of work done by Henke and Bienen (2013, 2014) and Henke and Grabe (2008), the effect of various installation methods such as quasi-static jacking, impact and vibratory driving on soil plugging, a phenomenon where the soil goes up inside the tubular pile and increases the penetration resistance, were both numerically and experimentally evaluated. They concluded that the driving method affects the soil behavior in terms of the formed soil plug inside the pile.

Heins et al. (2016) simulated the vibratory pile installation using the so-called zipper modeling technique, where a very small tube is defined inside the soil and on the pile axis which is initially in contact with soil. During the penetration, the soil looses its contact with the tube and starts to interact with the pile. Despite handling the induced large deformation due to pile penetration, this technique is reported to have some drawbacks regarding soil drifting and twisting (S. Henke 2010).

Different installation frequencies and driving energies were applied to the pile. Results consisted excess pore water pressure values as well as the resulting penetration depth. It was concluded that by applying more frequency and low energy, more penetration is achieved.

In a recent study done by Qin et al. (2017), numerous field tests were compiled to assess the effect of seven influencing parameters in the vibratory driving system on penetration rate of three different sheet pile sections. Results suggest that the effect of vibratory driving becomes significant at later stages of the penetration.

The structure of this paper is as follows. First, the theoretical background of the numerical model considerations including the chosen element formulation and the soil material model are presented. Then, the developed numerical model is described. The model is validated against an experiment followed by a parametric study on frequency effects on the surrounding soil. Subsequently, the obtained results from the parametric study are presented and discussed. Concluding remarks and outlook on future works are given at the end.

\section{THEORETICAL BACKGROUND}

This section gives a brief description of the utilized material model, the numerical approach, and the contact formulation used to simulate the soil-structure interaction.

\section{The hypoplastic material model for granular materials}

The hypoplastic material model developed by von Wolffersdorff (1996) and improved by Niemunis and Herle (1997) is used in this study. Generally, the idea behind the hypoplasticity concept is that it does not distinguish between elastic and plastic deformation (Kolymbas 1977). However, the first developed models exhibited shortcomings in capturing the realistic soil behavior under cyclic loading. The strain accumulated at each load cycle, resulting in a very significant strain accumulation 
compared to the experimental measurements. This issue was addressed by an intergranular strain tensor introduced as an additional state variable which represents the strain history (Niemunis and Herle 1997).

Unlike elastoplastic material models, the hypoplastic constitutive model is defined by a single incrementally nonlinear equation. The stress rate of the granular material, $\dot{\mathrm{T}}$, is determined by the effective stress, T, intergranular strain, $\delta$, and the void ratio, e (Niemunis and Herle 1997):

$$
\dot{\mathbf{T}}=\mathcal{M}(\mathbf{T}, \mathrm{e}, \delta): \mathbf{D}
$$

The void ratio in the Eq. (1) is constrained by $e_{i}$ and $e_{d}$ which reflect the minimum and maximum void ratio, respectively. Another limiting parameter is the void ratio at the critical state, $e_{c}$. To adapt the limiting void ratio parameters based on the current mean pressure a granular hardness parameter, $\mathrm{h}_{\mathrm{s}}$, is defined.

Hypoplasticity predicts the nonlinear and inelastic behavior of granular materials such as dilatancy, owing to its dependency on the void ratio of the soil. Due to its robustness observed in previous works, this material model is also used in the present study.

\section{The MMALE numerical approach}

Problems involving large soil deformation are often inevitable in geotechnical engineering. For instance, pile penetration, slope failures and avalanches, and liquefaction are major areas of interest during the last decades (Aubram et al. 2017). Numerical analysis of such problems is challenging, particularly due to the complex material behavior. The Finite Element Method (FEM) can be generally considered as a suitable numerical approach to address these problems. In FEM, there are two principal viewpoints to treat material deformations, namely the Lagrangian viewpoint and the Eulerian viewpoint.

In the Lagrangian viewpoint, the computational grid is pinned to the material particles, meaning that if the soil deforms, the grid deforms accordingly. In this approach considerable shortcomings arise when the soil significantly deforms, i.e. elements may encounter large distortion which leads to the solution divergence or unreliable results (Belytschko et al. 2000). On the other hand, in the Eulerian viewpoint, the grid is fixed and the material moves freely through the grid. Although large deformations and vorticity issues are addressed, the Eulerian approach requires extra considerations for treating path-dependent material behavior and tracking material interfaces (Benson 1992).

A new class of methods achieved to combine these two approaches to gather the advantages of both viewpoints while minimizing their shortcomings. Considering the grid-based methods, the most promising approaches include the Simplified or SingleMaterial Arbitrary Lagrangian-Eulerian (SALE), Coupled Eulerian-Lagrangian (CEL), and Multi-Material Arbitrary Lagrangian-Eulerian (MMALE) methods (Benson 1992). In SALE, remeshing/rezoning after each Lagrangian step is performed. In a comprehensive work done by Aubram $(2013,2015)$ this method is thoroughly discussed and evaluated. Bakroon et al. (2017) studied the achieved performance in numerical calculations by using SALE, compared to the Lagrangian explicit and implicit methods 
The efficiency of SALE method in the aspect of both accuracy and mesh improvement was proved for problems associated with moderate deformation. However, for large deformation, this method still holds drawbacks. This drawback is caused by the material boundary/interfaces which can only slide along the material boundary/interface. For a more detailed discussion the reader is referred to (Aubram et al. 2017; M. Bakroon et al. 2018b)

Therefore, alternative methods such as CEL and MMALE have been developed which both employ a non-aligned grid with material boundaries and interfaces. Thus, some grid elements may contain a mixture of two or more materials which is referred to as multi-material elements. In both methods, a material-free or void zone must be defined within the grid holding neither mass nor strength such that the materials can flow into these regions of the physical space. In both CEL and MMALE, after performing one or several Lagrangian steps, the mesh is rezoned to its initial configuration to maintain mesh quality (rezoning/remeshing step). In case of MMALE, a new arbitrary mesh is developed which is different from the initial mesh configuration. Subsequently, the solution is transported from the deformed mesh to the updated or original mesh (remapping/advection step). Fig. 1 illustrates the differences between the aforementioned methods. The sub-steps are not performed simultaneously but in series using the operator-splitting technique (Benson 1992).

A recent study conducted by Bakroon et al. (2018b) assessed the feasibility of CEL and MMALE methods in realistic geotechnical large deformation problems in comparison with SALE and classical Lagrangian methods. It was concluded that MMALE and CEL can be considered as promising candidates for solving complex large deforming problems. The applicability of MMALE in conjunction with a complex soil material model was investigated in another work done by Bakroon et al. (2018a).

\section{Pile-soil interaction}

To define the interaction between the pile and the soil, a robust contact scheme should be introduced. Usually, in CEL and MMALE formulation, the structural part and the soil are defined as the Lagrangian and Eulerian part, respectively. Penalty contact is generally employed due to its simplicity and robustness. The interaction between Lagrangian and Eulerian nodes is modeled as springs whose seeds and anchors are attached to the Lagrangian and Eulerian nodes, respectively (Benson and Okazawa 2004).

A schematic view of the penalty contact scheme is shown in Fig. 2 where two springs for both normal and tangential directions ( $\mathrm{k}_{\mathrm{t}}$ and $\mathrm{k}_{\mathrm{n}}$, respectively) are defined. The contact forces are calculated by considering a small amount of penetration, using the equation $F_{p}=k_{p} d_{p}$, where $d_{p}$ is the penetration depth, $F_{p}$ is the spring force consisting of two principal forces, $\mathrm{f}_{\mathrm{n}}$, and $\mathrm{f}_{\mathrm{t}}$ for normal and tangential direction, respectively (Benson and Okazawa 2004). 


\section{NUMERICAL MODEL}

\section{Description of the model}

In this section, a description of modeling considerations using MMALE technique in LS-DYNA/Explicit is presented where a pile is installed in the soil using a vibratory force. An axisymmetric model is developed to reduce the computational costs. The model configuration is shown in Fig. 3a. The load history curve of the vibratory force is depicted in Fig. 3b.

The rigid pile has $0.5 \mathrm{~m}$ height, $0.2 \mathrm{~m}$ diameter, and $0.005 \mathrm{~m}$ thickness which is modeled using the conventional 2D Lagrangian shell element formulation with reduced integration point and a uniform element size of $0.005 \mathrm{~m}$.

For the soil, a mesh with $2 \mathrm{~m}$ height and $1 \mathrm{~m}$ radius with the one-point integration MMALE shell element formulation is generated. The equipotential smoothing technique is applied to the mesh nodes to maintain the mesh quality (Winslow 1963). For the advection step, the $2^{\text {nd }}$ order accurate van Leer method is chosen (Van Leer 1977).

An unstructured mesh, ranging from $0.005-0.05 \mathrm{~m}$ element width is used. The mesh contains the soil up to the height of $1.8 \mathrm{~m}$. A void domain with $0.2 \mathrm{~m}$ height, which has neither mass nor strength, is defined above the soil material to enable the soil to move to this domain after penetration starts. The hypoplastic material model is adopted, whose corresponding material constants for Berlin sand are listed in Table 1. The relative density of the soil is $I_{D}=75 \%\left(e_{\text {initial }}=0.465\right)$. The initial stress in the soil is defined with assigning the gravity acceleration as $10 \mathrm{~m} / \mathrm{s}^{2}$ and using the lateral earth pressure, $\mathrm{K}_{0}=0.5$. To define the coupling between pile and soil, penalty contact is defined with a tangential friction coefficient of 0.2 which corresponds to $\tan (\phi / 3)$. The pile is fixed against horizontal movements. The lateral sides of the soil are constrained against movements in a direction perpendicular to their faces, while fixity in all directions is applied to the bottom of the soil.

\section{Validation against experimental results}

An experimental test was conducted at the laboratory of the Chair of Soil Mechanics and Geotechnical Engineering at Technische Universität Berlin (TU Berlin). Details regarding the test set-up can be found in the published work done by Le et al. (2018). The experiment includes a half-cylindrical pile with $1.5 \mathrm{~m}$ length, $0.005 \mathrm{~m}$ thickness, and $0.2 \mathrm{~m}$ outer diameter placed in a container filled with the Berlin sand and consists of three rigid steel walls and one glass panel. The pile movement is constrained in the horizontal direction using pile guides. A vibratory motor is installed on the pile head with $1670 \mathrm{~N}$ driving force and $23 \mathrm{~Hz}$ frequency. The imposed dead load on the pile is about $410 \mathrm{~N}$. To measure pile penetration, two displacement sensors are installed on the pile.

Fig. 4 shows the comparison of the resulting displacement curve from the numerical model compared against experimental measurement. At early stages of driving, the penetration rate is significant due to the less soil resistance and confining pressure. Afterward, the frictional force increases since the resulting force normal to the pile skin increase considerably, causing the penetration rate to decrease. 
In Fig. 5a and Fig. 5b, the velocity vectors at two-time stamps are shown which corresponds to the times when the pile pushes the soil and when the pile is pulled out (see Fig. 5c). During the push as shown in Fig. 5a, the soil particles under the pile tip tend to move to lateral sides and then to the upward direction. On the other hand, the particles near the pile shaft are moved with the pile due to the friction. The soil motion inside the pile (i.e. the left side of the pile in the model) is more significant than outside the pile. This can be argued to be caused by the more confinement stress inside the pile. Moreover, the soil motion inside the pile is rather uniform, which seems like that the soil moves as a block according to the pile motion. This point is mentioned in several works in literature and is referred to as the "soil plugging", a phenomenon where the soil inside the pile moves as a block along with pile during the penetration (De Nicola and Randolph 1997). During the pile pull out stage as shown in Fig. 5b, the soil movement direction reverse as the pile tends to go up. Again, the soil inside the pile moves as a block with a more uniform velocity than the soil outside the pile, but with less magnitude compared to the pushing stage. The reversed movement is caused by releasing the pressure resulted by the pile push in the previous stage. Hence, the soil tends to return to its previous and more stable state which is similar to what is observed in loading-unloading tests. Concerning the points discussed above, it can be argued that the numerical model captures reasonably the soil condition in the experiment.

\section{Parametric study}

To study the effect of vibration on the driving performance, six models with various frequencies are developed and compared. Both the static and dynamic load amplitudes are maintained for all models while the frequencies are changed to 12, 16, 19, 23, 27 and $30 \mathrm{~Hz}$. All other considerations including model geometry and element size, initial state and boundary conditions are also maintained as those used in the previous section. The penetration curves for all models are plotted against time in Fig. 6 . The difference between the penetration trends becomes significant after about 4 seconds which corresponds to approximately $0.3 \mathrm{~m}$. It may be argued that the soil at the lower depth did not show a high resistance against penetration. Therefore, this part of the penetration can be mainly attributed to the force induced by the static load.

It is observed that the trend and the final value of the penetration is identical for lower frequencies of 12, 16, and $19 \mathrm{~Hz}$. In addition, the models with 23 and $30 \mathrm{~Hz}$ resulted in a more penetration value than those with lower frequencies. Finally, the model with 27 $\mathrm{Hz}$ gave the most penetration depth.

During the vibratory installation, the soil is affected in several ways. First, the area around the pile is partly loosened due to the disturbance enforced by the pile vibration. This makes the pile penetration easier since the disturbed area exhibits lower resistance. Second, the soil regime located further from the pile is densified due to the same reason, making the soil grains to reach a more compacted arrangement. This might adversely affect the driving performance since the compacted soil shows more confinement and resistance, which leads to less penetration. Hence, an optimized frequency shall be assigned which results in more penetration without significant resistance.

To assess the compaction and loosening, the void ratio contours are shown in Fig. 7. It can be observed that by increasing the frequency, the compacted soil volume increases 
as well. On the contrary, the growth of the loosened soil regime is not as significant as the densified area. Thus, by using a higher frequency a larger area is compacted which resists more against the penetration and therefore reduces the disturbance effect which facilitated the penetration. Consequently, using the higher frequency may not always result in a more penetration depth.

In the problem under study, this issue is noticed for the model with the frequency of 30 , i.e. a less penetration is achieved for the model with the frequency of $30 \mathrm{~Hz}$ compared to the model with the frequency of $27 \mathrm{~Hz}$.

Fig. 8 shows the lateral stress distribution in the soil after 5 seconds of pile driving. The lateral stress is important in defining the soil plugging. The investigation of the plugging is important since it contributes to the pile bearing capacity, yet increases soil resistance against pile driving. The influenced area varies according to the pile frequency, i.e. with more frequency, a larger area is affected. The stress distribution near the pile is noisy which can be attributed to the soil disturbance.

To study the difference in the lateral stress values inside and outside the pile during driving, the induced lateral forces curves on the pile are plotted in Fig. 9. For higher frequency ranges, a significant increase in stress values inside the pile occurs after about 4 seconds, which is the similar time stamp as when the differences in penetration curves appeared. This slope change is argued to be related to the plugging initiation (Henke and Bienen 2013). For lower frequency ranges, a small difference is seen, meaning that the soil lateral stress inside and outside the soil are pretty identical.

\section{CONCLUSIONS}

In this study, a numerical model was developed using the MMALE method in conjunction with a hypoplastic material model to investigate the effects of the frequency magnitude on the evolution of soil stress and density (void ratio) during the vibratory installation of a tubular pile. The penetration depth of the numerical model was compared to the experimental measurement to validate the model. Afterward, a parametric study was performed where the applied frequency in the vibratory load was changed to study soil behavior.

The resulting penetration depths were first compared. At early stages of the penetration, no significant differences were observed since the static load was dominating, making the frequency load effects negligible. Frequency effects become more apparent at large penetration depths. Due to the vibratory driving, the soil regime near the pile is disturbed, which reduces the soil resistance and thus results in more penetration. In addition, by raising the frequency value, more penetration was observed until reaching the value of $27 \mathrm{~Hz}$. Values more than $27 \mathrm{~Hz}$ resulted in less penetration.

To study the underlying reason for less penetration, void ratio and lateral stresses were assessed. It was concluded that by using more frequency, more area is densified, which makes the soil stiffer. Therefore, more resistance is observed against driving. Concerning the simultaneous effect of soil disturbance and the densification on pile driving, it is concluded that an optimum frequency range should be determined to ensure the maximum penetration using the same load magnitude.

The employed numerical model used in this study can be used in further investigations such as pile driving in an undrained condition. Besides, other forms of driving methods can be compared against each other to reach a suitable pile performance. 


\section{ACKNOWLEDGMENTS}

The authors are thankful for the partial financial support obtained from German Academic Exchange Service (DAAD) with grant number 91561676.

\section{REFERENCES}

Aubram, D. (2013). An arbitrary Lagrangian-Eulerian method for penetration into sand at finite deformation. Shaker Verlag, Aachen, Berlin, Germany.

Aubram, D. (2015). Development and experimental validation of an arbitrary Lagrangian-Eulerian (ALE) method for soil mechanics. Geotechnik, 38(3), 193204. doi:10.1002/gete.201400030

Aubram, D., Rackwitz, F., \& Savidis, S. A. (2017). Contribution to the non-lagrangian formulation of geotechnical and geomechanical processes. In Lecture Notes in Applied and Computational Mechanics (Vol. 82, pp. 53-100). Springer International Publishing. doi:10.1007/978-3-319-52590-7_3

Bakroon, M., Daryaei, R., Aubram, D., \& Rackwitz, F. (2017). Arbitrary LagrangianEulerian Finite Element Formulations Applied to Geotechnical Problems. In J. Grabe (Ed.), Numerical Methods in Geotechnics (pp. 33-44). Hamburg, Germany: BuK! Breitschuh \& Kock GmbH. doi:978-3-936310-43-6

Bakroon, M., Daryaei, R., Aubram, D., \& Rackwitz, F. (2018a). Implementation and Validation of an Advanced Hypoplastic Model for Granular Material Behavior. In 15th International LS-DYNA® Users Conference (p. 12). Detroit, Michigan, USA.

Bakroon, M., Daryaei, R., Aubram, D., \& Rackwitz, F. (2018b). Multi-Material Arbitrary Lagrangian-Eulerian and Coupled Eulerian-Lagrangian methods for large deformation geotechnical problems. In C. Sagaseta (Ed.), Numerical Methods in Geotechnical Engineering (NUMGE 2018) (p. 8). Porto, Portugal.

Belytschko, T., Liu, W. K., \& Moran, B. (2000). Nonlinear Finite Elements For Continua And Structures. Advances in cancer research (Vol. 104). Chichester: John Wiley. doi:10.1016/S0065-230X(09)04001-9

Benson, D. J. (1992). Computational methods in Lagrangian and Eulerian hydrocodes. Computer Methods in Applied Mechanics and Engineering, 99(2-3), 235-394. doi:10.1016/0045-7825(92)90042-I

Benson, D. J., \& Okazawa, S. (2004). Contact in a multi-material Eulerian finite element formulation. Computer Methods in Applied Mechanics and Engineering, 193(39-41 SPEC. ISS.), 4277-4298. doi:10.1016/j.cma.2003.12.061

De Nicola, A., \& Randolph, M. F. (1997). The plugging behaviour of driven and jacked piles in sand. Géotechnique, 47(4), 841-856. doi:10.1680/geot.1997.47.4.841

Feng, R., \& Deschamps, R. j. (2000). A Study of the Factors Influencing the Penetration and Capacity of Vibratory Driven Piles. SOILS AND FOUNDATIONS, 40(3), 43-54. doi:10.3208/sandf.40.3_43

Heins, E., Hamann, T., Grabe, J., \& Hannot, S. (2016). Numerical investigation of the influence of the driving frequency during pile installation of tubular piles. Geotechnik, 39(2), 98-109. doi:10.1002/gete.201600014 
Henke, S. (2010). Influence of pile installation on adjacent structures. International Journal for Numerical and Analytical Methods in Geomechanics, 34(11), 11911210. doi:10.1002/nag.859

Henke, S., \& Bienen, B. (2013). Centrifuge tests investigating the influence of pile cross-section on pile driving resistance of open-ended piles. International Journal of Physical Modelling in Geotechnics, 13(2), 50-62. doi:DOI 10.1680/ijpmg.12.00012

Henke, S., \& Bienen, B. (2014). Investigation of the influence of the installation method on the soil plugging behaviour of a tubular pile. ICPMG2014 - Physical Modelling in Geotechnics, 2(1995), 681-687. doi:10.1201/b16200-94

Henke, S., \& Grabe, J. (2008). Numerical investigation of soil plugging inside openended piles with respect to the installation method. Acta Geotechnica, 3(3), 215223. doi:10.1007/s11440-008-0079-7

Holeyman, A., \& Whenham, V. (2017). Critical Review of the Hypervib1 Model to Assess Pile Vibro-Drivability. Geotechnical and Geological Engineering, 35(5), 1933-1951. doi:10.1007/s10706-017-0218-8

Kolymbas, D. (1977). A rate-dependent constitutive equation for soils. Mechanics Research Communications, 4(6), 367-372. doi:10.1016/0093-6413(77)90056-8

Le, V. H., Remspecher, F., \& Rackwitz, F. (2018). Influence of installation effects on the cyclic behaviour of monopile foundation for offshore wind power turbine. In M. Randolph \& K. H. Pham (Eds.), Submitted to "The First Vietnam Symposium on Advances in Offshore Engineering” (p. 11). Hanoi, Vietnam.

Niemunis, A., \& Herle, I. (1997). Hypoplastic model for cohesionless soils with elastic strain range. Mechanics of Cohesive-frictional Materials, 2(4), 279-299.

Qin, Z., Chen, L., Song, C., \& Sun, L. (2017). Field Tests to Investigate the Penetration Rate of Piles Driven by Vibratory Installation. Shock and Vibration, 2017. doi:10.1155/2017/7236956

Rausche, F. (2002). Modeling of vibratory pile driving. Proceedings of Vibratory Pile Driving and Deep Soil Compaction: TRANSVIB2002, 12.

Van Leer, B. (1977). Towards the ultimate conservative difference scheme. IV. A new approach to numerical convection. Journal of Computational Physics, 23(3), 276-299. doi:10.1016/0021-9991(77)90095-X

von Wolffersdorff, P.-A. (1996). A hypoplastic relation for granular materials with a predefined limit state surface. Mechanics of Cohesive-Frictional Materials, 1(3), 251-271. http://doi.wiley.com/10.1002/\%28SICI\%2910991484\%28199607\%291\%3A3\%3C251\%3A\%3AAID-CFM13\%3E3.0.CO\%3B2-3

Winslow, A. M. (1963). Equipotential zoning of two-dimensional meshes (UCRL7312). United States.

\section{TABLES}

Table 1 Hypoplastic material constants for Berlin sand

\begin{tabular}{|c|c|c|c|c|c|c|c|c|c|c|c|c|}
\hline$\varphi_{\mathrm{c}}\left[{ }^{\circ}\right]$ & $h_{\mathrm{s}}[\mathrm{MPa}]$ & $n$ & $e_{\mathrm{d} 0}$ & $e_{\mathrm{c} 0}$ & $e_{\mathrm{i} 0}$ & $\alpha$ & $\beta$ & $m_{R}$ & $m_{T}$ & $R$ & $\chi$ & $\beta_{r}$ \\
\hline 31.5 & $230^{*}$ & 0.3 & 0.391 & 0.688 & 0.791 & 0.13 & 1 & 4.4 & 2.2 & $1 \times 10^{-4}$ & 6.0 & 0.2 \\
\hline
\end{tabular}

*The actual value of granular hardness, $\mathrm{h}_{\mathrm{s}}$, is $2300 \mathrm{MPa}$. This value is reduced by $10 \%$ due to low-stress soil state 


\section{FIGURES}

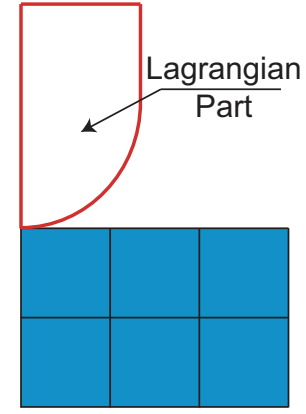

Initial configuration

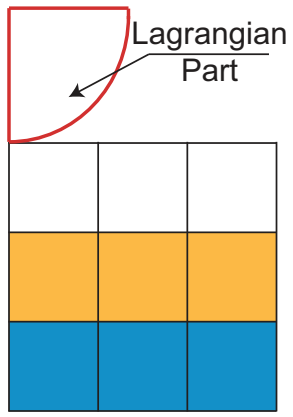

Initial configuration

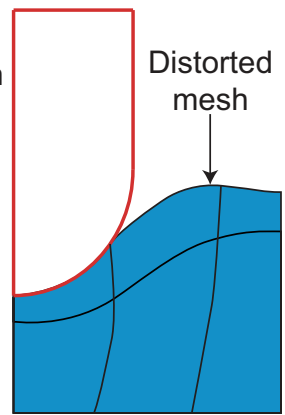

Lagrangian

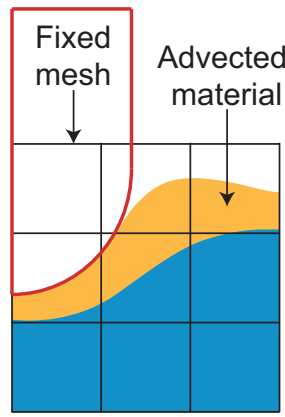

CEL

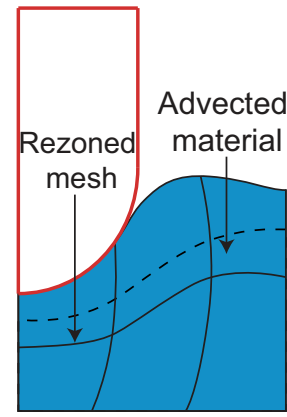

SALE

Material A

Void elements

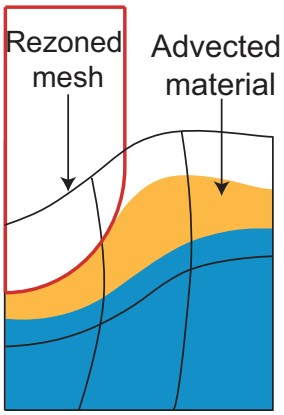

MMALE

Material B $\square$ Lagrangian part

Fig. 1. Schematic diagram of CEL, SALE, and MMALE approaches comparing the effect of mesh rezoning (remeshing) and remapping (advection) steps of the solution.

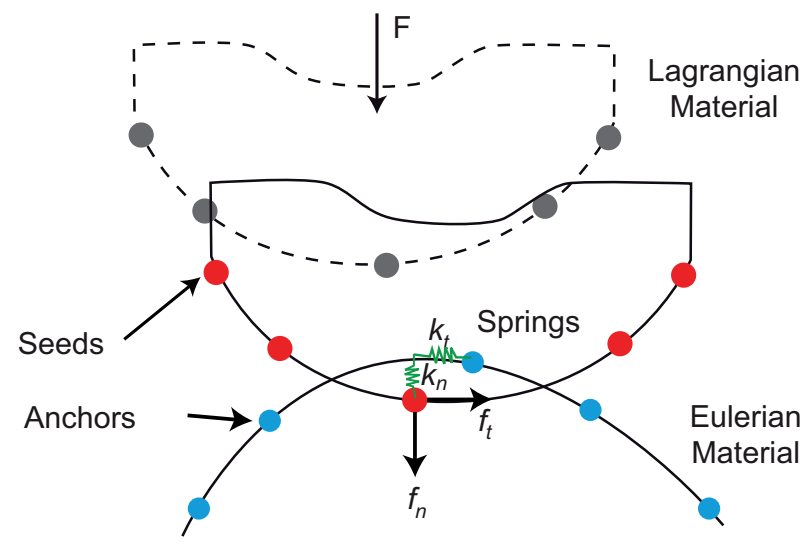

Fig. 2 Schematic view of penalty contact scheme 

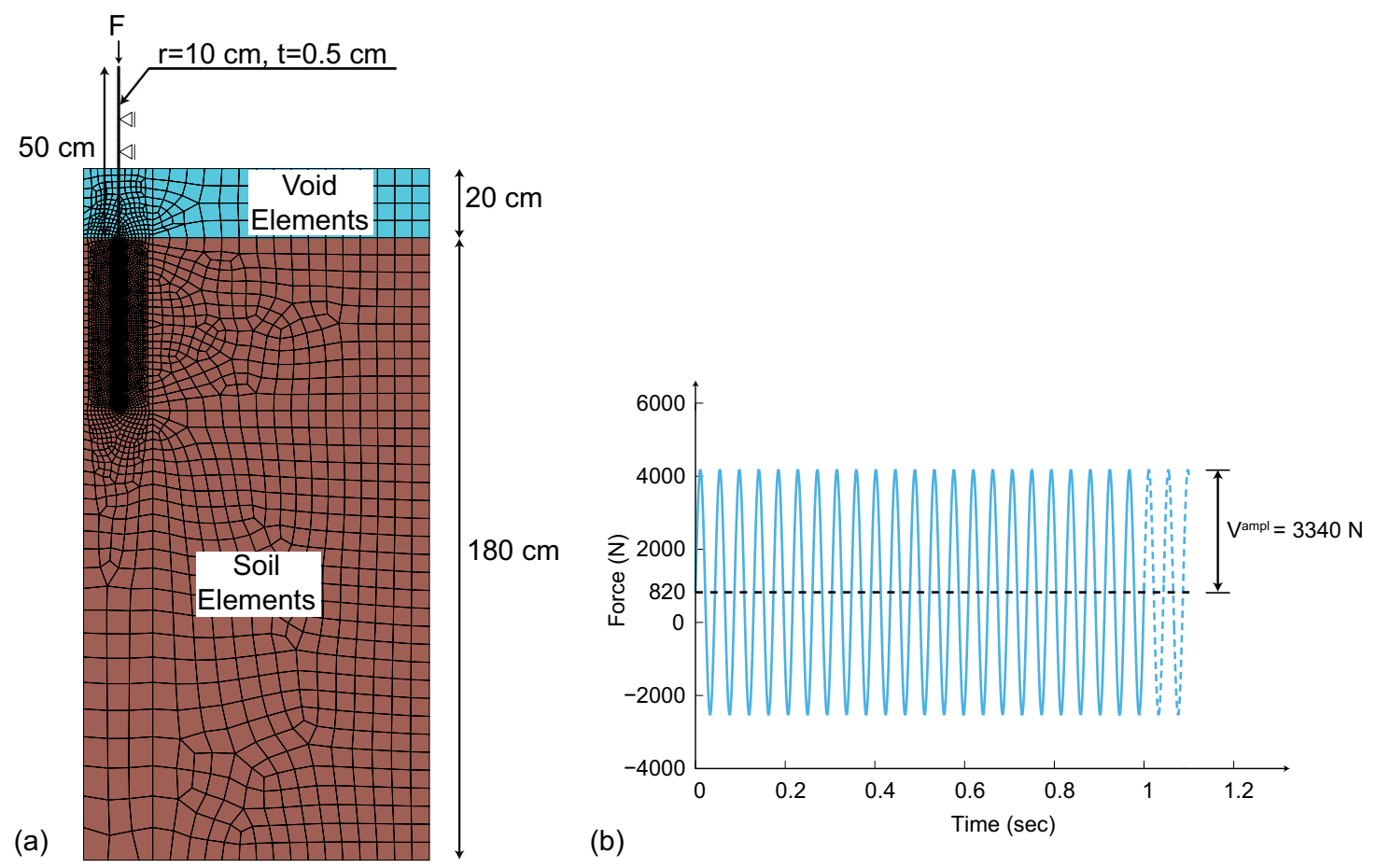

Fig. 3. Schematic diagram of the axisymmetric numerical model configuration

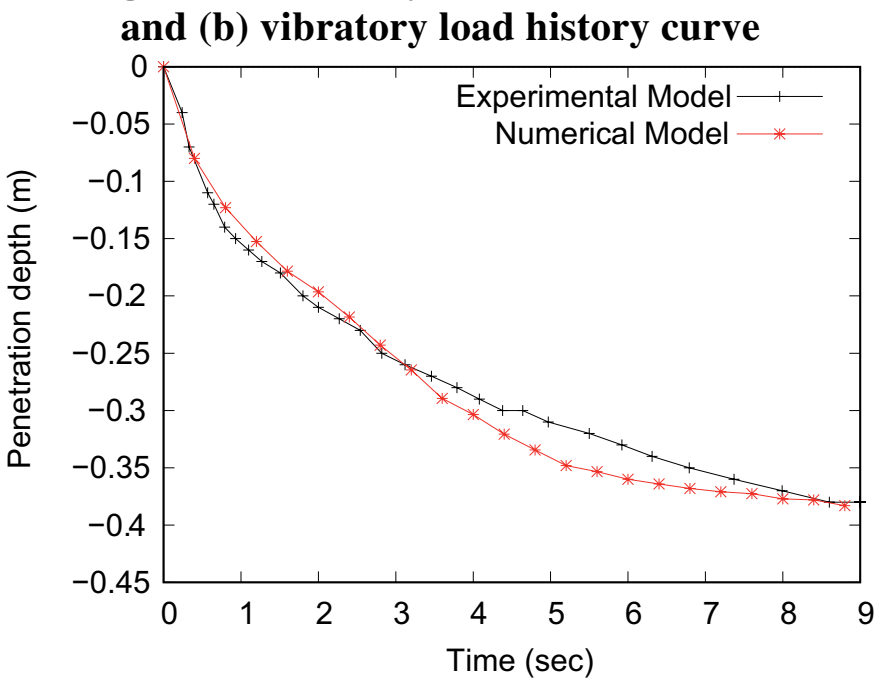

Fig. 4. Penetration depth vs. time curve obtained from numerical model and experimental measurement 

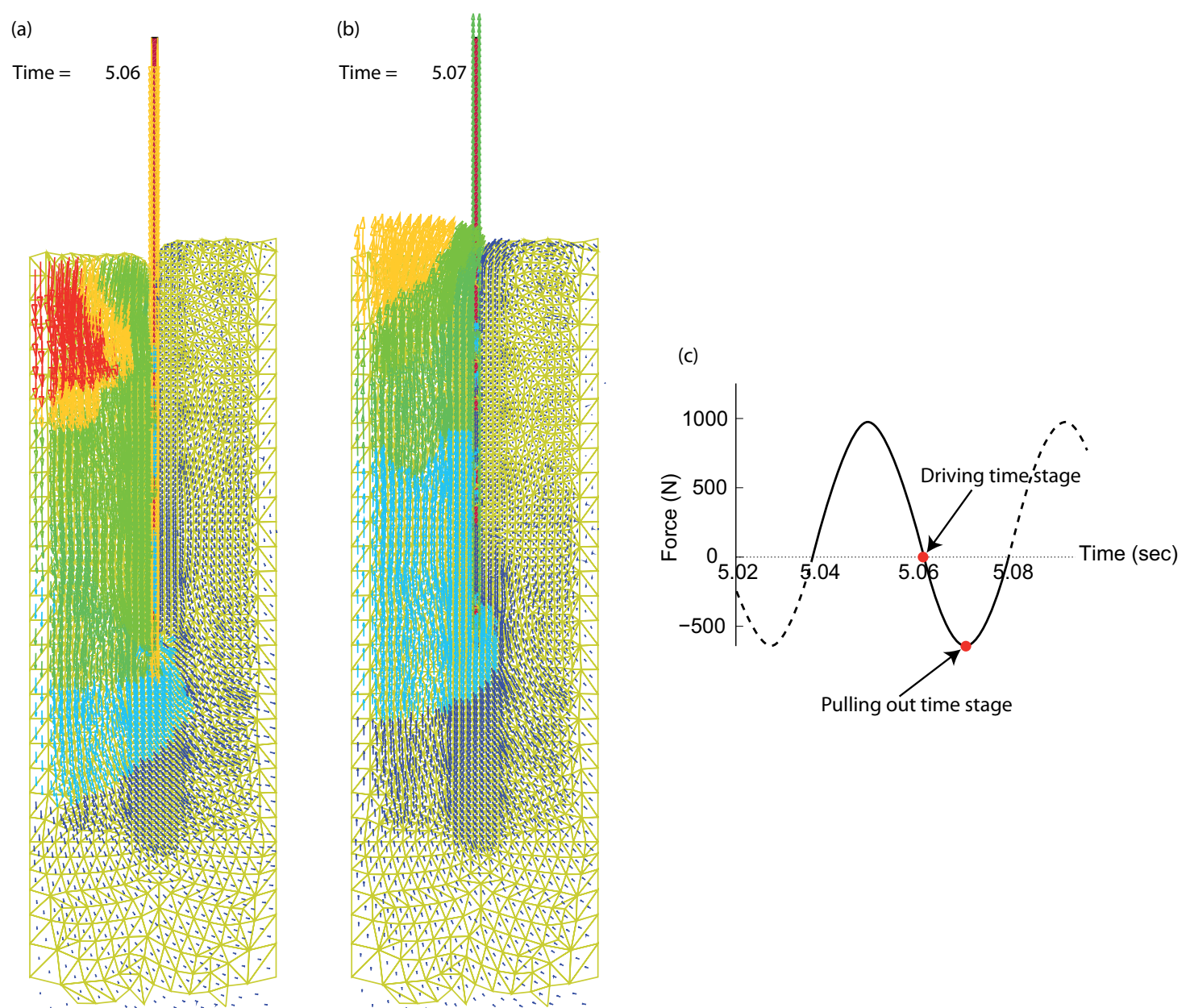

Fig. 5. Velocity vector of the soil regime during pile (a) driving, (b) pulling out and (c) the corresponding loading time stages for the validation model

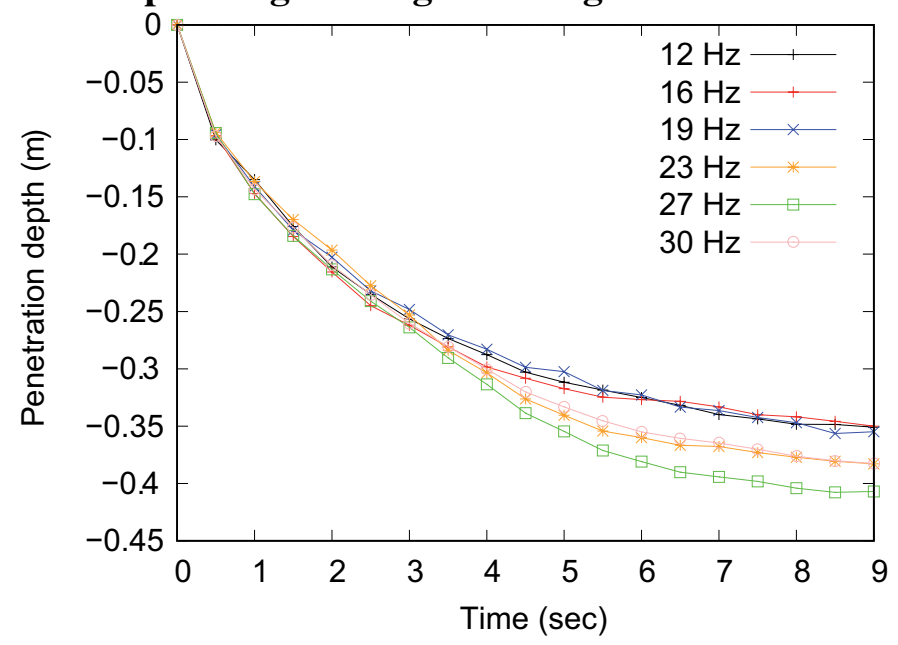

Fig. 6. Penetration curves of the models with different frequencies versus time 


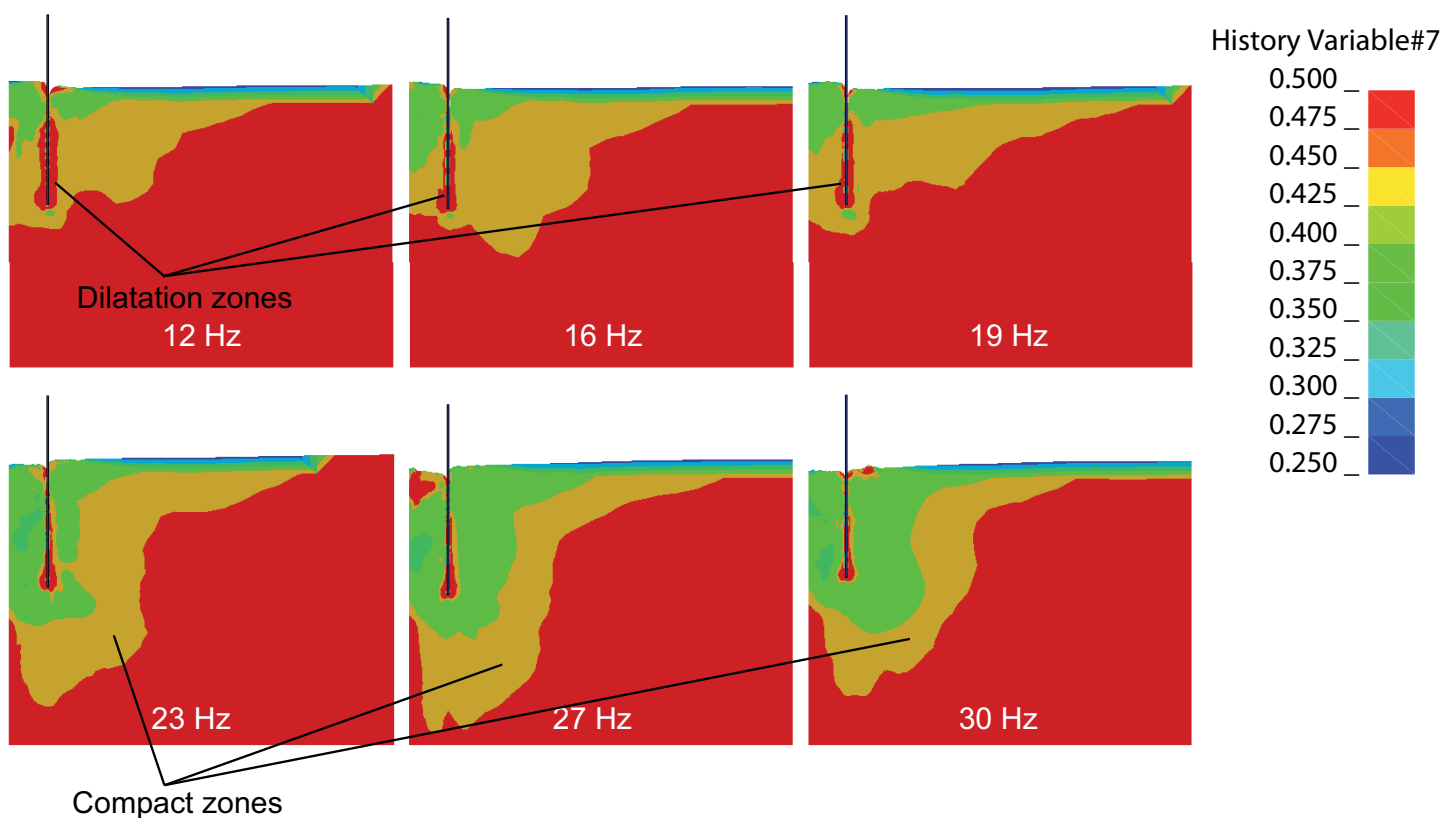

Fig. 7. Void ratio distribution after $t=5 \mathrm{~s}$ of pile driving corresponding to different frequencies (initial void ratio, $e_{\text {initial }}=\mathbf{0 . 4 6 5}$ ) 


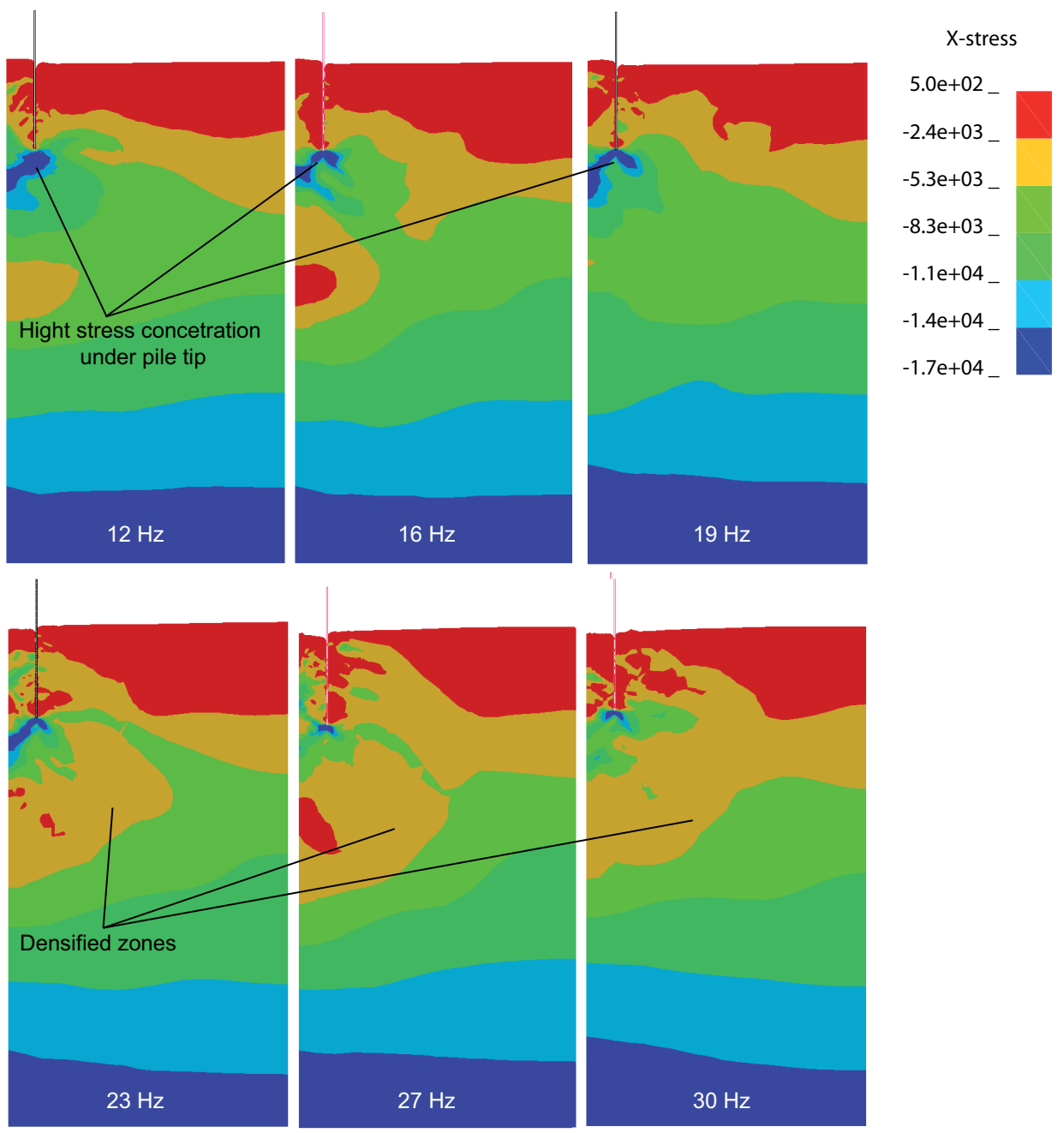

Fig. 8. Horizontal stress distribution after $t=5 \mathrm{~s}$ of pile driving corresponding to different frequencies

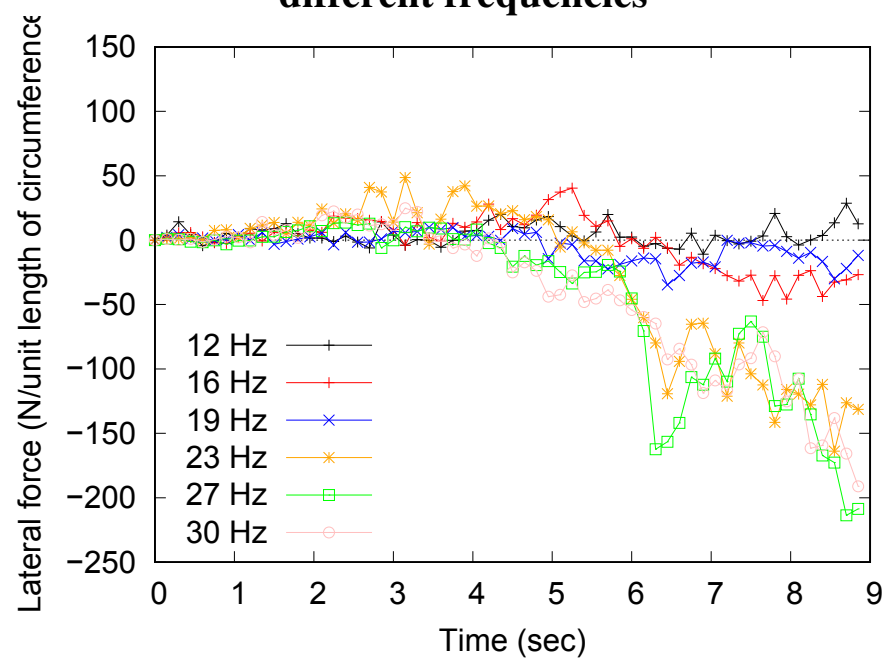

Fig. 9. Total radial force distribution in the whole pile length corresponding to different frequencies (the negative value indicate larger internal than external soil radial stress) 